\title{
Signal Shaping for Generalized Spatial Modulation and Generalized Quadrature Spatial Modulation
}

\author{
Shuaishuai Guo, Member, IEEE, Haixia Zhang, Senior Member, IEEE, Peng Zhang, Member, IEEE, \\ Shuping Dang, Member, IEEE, Cong Liang, and Mohamed-Slim Alouini, Fellow, IEEE
}

\begin{abstract}
This paper investigates generic signal shaping methods for multiple-data-stream generalized spatial modulation (GenSM) and generalized quadrature spatial modulation (GenQSM). Three cases with different channel state information at the transmitter (CSIT) are considered, including no CSIT, statistical CSIT and perfect CSIT. A unified optimization problem is formulated to find the optimal transmit vector set under size, power and sparsity constraints. We propose an optimizationbased signal shaping (OBSS) approach by solving the formulated problem directly and a codebook-based signal shaping (CBSS) approach by finding sub-optimal solutions in discrete space. In the OBSS approach, we reformulate the original problem to optimize the signal constellations used for each transmit antenna combination (TAC). Both the size and entry of all signal constellations are optimized. Specifically, we suggest the use of a recursive design for size optimization. The entry optimization is formulated as a non-convex large-scale quadratically constrained quadratic programming (QCQP) problem and can be solved by existing optimization techniques with rather high complexity. To reduce the complexity, we propose the CBSS approach using a codebook generated by quadrature amplitude modulation (QAM) symbols and a low-complexity selection algorithm to choose the optimal transmit vector set. Simulation results show that the OBSS approach exhibits the optimal performance in comparison with existing benchmarks. However, the OBSS approach is impractical for large-size signal shaping and adaptive signal shaping with instantaneous CSIT due to the demand of high computational complexity. As a low-complexity approach, CBSS shows comparable performance and can be easily implemented in large-size systems.
\end{abstract}

Index Terms-Multiple-input multiple-output, generalized spatial modulation, generalized quadrature spatial modulation, signal shaping, precoding, maximizing the minimum Euclidean distance, sparsity constraint

\section{INTRODUCTION}

$\mathbf{M}$ ULTIPLE-DATA-STREAM generalized spatial modulation (GenSM) and generalized quadrature spatial modulation (GenQSM) have emerged as new techniques for multiple-input multiple-output (MIMO) communications with reduced radio frequency $(\mathrm{RF})$ chains and fast antenna switches. As generalized forms of spatial modulation (SM) [1]-[4] and quadrature spatial modulation (QSM) [5]-[7], they employ

S. Guo, S. Dang and M.-S. Alouini are with Computer Electrical and Mathematical Sciences \& Engineering (CEMSE) Division, King Abdullah University of Science and Technology (KAUST), Thuwal 239556900, Kingdom of Saudi Arabia. (e-mail: \{shuaishuai.guo; shuping.dang; slim.alouini\}@kaust.edu.sa).

H. Zhang and C. Liang are with Shandong Provincial Key Laboratory of Wireless Communication Technologies, Shandong University, Jinan 250061, China, (e-mail: haixia.zhang@sdu.edu.cn; congliang@mail.sdu.edu.cn).

P. Zhang is with the School of Computer Engineering, Weifang University, Weifang 261061, China (e-mail: sduzhangp@163.com). multiple in-phase and quadrature (IQ) RF chains for multipledata-steam transmission and additionally carry information by the selection of transmit antenna combinations (TACs). By varying the number of RF chains, they can achieve an attractive trade-off between spectral efficiency (SE) and energy efficiency (EE). For general $\left(N_{t}, N_{r}, N_{R F}, n\right)$ GenSM/GenQSM MIMO systems with $N_{t}$ transmit antennas and $N_{r}$ receive antennas conveying a fixed length of $n$-bit stream via $N_{R F} \mathrm{RF}$ chains, this paper investigates generic signal shaping methods to find the optimal $2^{n}$ transmit vectors. This is a rather intricate task because it couples the multi-dimensional signal constellation optimization as well as the spatial constellation optimization. In this paper, we aim to solve the problem based on the criterion maximizing the minimum Euclidean distance (MMED). After that, we also discuss the designs based on the criteria minimizing the symbol error rate (MSER) and maximizing the mutual information (MMI).

\section{A. Prior Work}

All existing signal constellation designs, spatial constellation designs, precoding schemes (including phase rotation and power allocation schemes) as well as their combinations can be viewed as signal shaping methods, because all of them affect the transmit vectors. Based on the required information, we classify prior work into the following two categories:

\section{1) Signal Shaping without CSIT}

Without CSIT, GenSM/GenQSM systems can benefit from the off-line design of signal and spatial constellation. Specifically, the analysis in [8] and [9] showed that the shape of the signal constellation greatly affects the error performance, based on which [10]-[14] investigated the signal constellation design for SM based on pre-defined constellation structures or optimization techniques. However, the assumption that all TACs utilize the same signal constellation in [10]-[14] limits the system performance improvement and the application to SM systems with an arbitrary number of antennas. To tackle this issue, the authors of [15] and [16] proposed an optimization strategy jointly considering the signal and spatial constellations for SM. [16] showed that the joint optimization strategy achieves better performance than the sole signal constellation optimization strategies. On the other hand, the gain is rather small yet costless, because the off-line design does not render any computational complexity for on-line data transmission. [16] extended the optimization strategy regarding both signal and spatial constellations in GenSM systems using inter-channel interference (ICI)-free single-data- 
stream transmission. However, the design for more spectralefficient multiple-data-stream GenSM was left unconsidered. Besides, little work is known on the constellation design for GenQSM other than [17] proposed a heuristic signal constellation optimization for QSM and [18] studied a latticecode-based constellation optimization strategy for GenQSM. These are based on predefined codebooks and can be viewed as an optimization strategy in the discrete space. Generic optimization strategy in the continuous complex field regarding both signal and spatial constellations for GenQSM remains unexplored.

2) Signal Shaping with CSIT

With instantaneous/statistical CSIT, GenSM/GenQSM systems can benefit from adaptive signal shaping. For instance, adaptive signal constellation design was investigated for SM in [19]; [20]-[22] studied antenna selection for SM systems, which can be regarded as the adaptive spatial constellation optimization; A large body of literature such as [23]-[38] probed into precoding schemes as well as their combinations with the adaptive signal or spatial constellation optimization. However, most literature considered SM or single-data-stream GenSM. To the best of our knowledge, few literature focused on the adaptive signal shaping for multiple-data-stream GenSM other than a recent work [38] that modifies a given multiple-dimensional signal constellation via a diagonal or full precoder for each TAC. However, such precoding-aided signal shaping methods in [38] are suboptimal. The reasons are twofolds: First, each TAC carries the same number of data symbol vectors in [38], while due to the random nature of wireless channels, different TACs corresponding to separate channels have distinct information-carrying capabilities; Second, the data symbol vectors in the signal constellation of an activated TAC are modified by the same precoder, which cannot guarantee the global signal shaping optimality, because the performance also highly depends on the previously given signal constellation. In addition, few work was dedicated to the adaptive shaping for GenQSM, except [6] that adjusts the precoding process for QSM systems with a single IQ RF chain.

In summary, both constellation design and adaptive signal shaping have been extensively investigated for SM and singledata-stream GenSM. A few of literature studied the signal shaping for QSM systems with a single IQ RF chain. However, the designs for multiple-data-stream GenSM/GenQSM with/without CSIT call for a systematic investigation, which motivates us to fill this gap with the contributions listed infra.

\section{B. Contributions}

- This paper formulates a unified signal shaping optimization problem for multiple-data-stream GenSM/GenQSM with/without CSIT based on the MMED criterion, which aims to find the optimal transmit vector set under a size constraint, a unit average power constraint and a special sparsity constraint.

- To solve the formulated problem, we reformulate the original problem to find the optimal signal constellations for each TAC. We optimize both the size and entry of all signal constellations. In particular, we suggest the use of a recursive design presented in [16] for the size optimization. The entry optimization is formulated as a large-scale non-convex quadratically constrained quadratic programming (QCQP) problem, which can be solved by existing optimization techniques with rather high computational complexity.

- To reduce the computational complexity and also to facilitate the implementation in realistic systems, we adopt a codebook generated by using quadrature amplitude modulation (QAM) symbols to generate all feasible transmit vectors. Then, we propose a low-complexity progressive selection algorithm to choose the optimal transmit vector set.

- For extension, we discuss the designs relying on the MSER and MMI criteria. Specifically, we formulate the signal shaping problems to minimize the upper bound on the symbol error rate (SER) and to maximize the lower bound on the mutual information, respectively. Analysis shows that both problems are reduced to the design based on the MMED criterion in the high signal-to-noise ratio (SNR) regime.

- Numerical comparisons with existing designs are presented to verify the superiority of our designs. In particular, we compare the proposed joint constellation design with the sole signal constellation design in open-loop systems without CSIT and closed-loop systems with statistical CSIT, where the well-recognized best signal constellations (e.g., the binary phase shift keying, BPSK) are included in comparisons; we compare the proposed adaptive signal shaping methods with precoding-aided signal shaping methods proposed in [38]. Comparison results show that the proposed optimization-based signal shaping (OBSS) approach considerably outperforms existing designs in literature and the proposed codebook-based signal shaping (CBSS) approach shows comparable performance with much lower complexity. Moreover, the performance of the proposed designs with imperfect channel state information (CSI) is also investigated by simulations.

\section{Organization}

The remainder of the paper is organized as follows. Section II describes the system model. Section III introduces the unified problem formulation for the signal shaping of multipledata-stream GenSM/GenQSM with/without CSIT based on the MMED criterion. In Sections IV and V, we introduce the OBSS and CBSS approaches, respectively. Sections VI discusses the extensions to the designs with MSER and MMI as optimization criteria. Numerical comparisons are presented in Section VII and conclusions are drawn in Section VIII.

\section{Notations}

In this paper, $a$ represents a scaler; $\mathbf{a}$ is a vector; $\mathbf{A}$ stands for a matrix. $\|\mathbf{a}\|_{0}$ and $\|\mathbf{a}\|_{2}$ stand for the $l_{0}$ norm and the $l_{2}$ norm of a, receptively. $\operatorname{diag}(\mathbf{a})$ is a diagonal matrix whose diagonal entries are from vector a. $\mathbf{a}(i)$ denotes the $i$ th entry of $\mathbf{a}$. $\operatorname{diag}\{\mathbf{A}\}$ stands for a vector formed by the diagonal elements of matrix $\mathbf{A}$. $\operatorname{det}(\mathbf{A})$ represents the determination of 


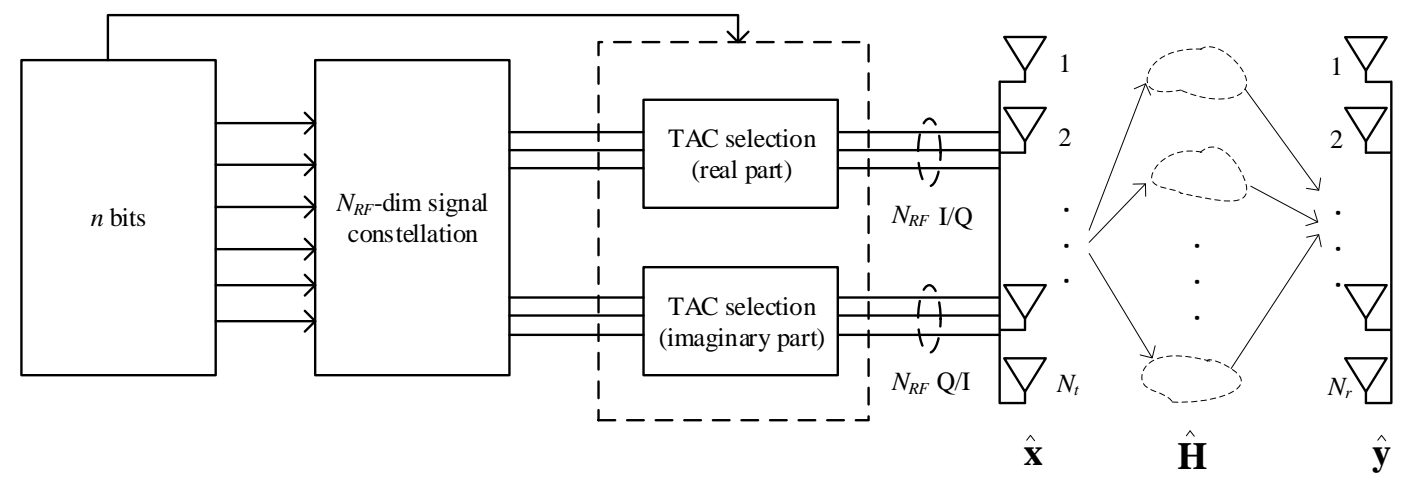

Fig. 1. A typical GenSM/GenQSM MIMO system.

matrix A. $\otimes$ stands for the Kronecker product. $\odot$ represents the Hadamard product. $(\cdot)^{T},(\cdot)^{\dagger}$ stand for the transpose and the conjugate, respectively. $\mathbb{R}$ is the real domain; $\mathbb{Z}$ represents the integer domain; $\mathbb{C}$ stands for the complex domain. $\mathcal{C N}\left(\mu, \sigma^{2}\right)$ denotes the complex Gaussian distribution with mean $\mu$ and variance $\sigma^{2} . \mathbb{E}_{\mathbf{A}}(\cdot)$ and $\mathbb{E}_{\mathbf{A}}[\cdot]$ represent the expectation operation with respect to $\mathbf{A}$. $\mathcal{A}$ is a set and $|\mathcal{A}|$ represents the size of set $\mathcal{A}$. $[\mathbf{A}]_{k, l}$ represents the $k$ th row $l$ th column entry of $\mathbf{A} . \Re(\cdot)$ and $\Im(\cdot)$ represent the real and imaginary parts, respectively. $\lfloor\cdot\rfloor$ represents the floor operation. $\left(\begin{array}{c}n \\ m\end{array}\right)$ is a binomial coefficient.

\section{System Model}

\section{A. System Framework}

In this paper, we consider an $\left(N_{t}, N_{r}, N_{R F}, n\right)$ GenSM/GenQSM MIMO system as illustrated in Fig. 1, where $N_{t}$ and $N_{r}$ represent the numbers of transmit and receive antennas; $N_{R F}$ is the number of RF chains and $n$ stands for the target transmission rate in bit per channel use (bpcu). Unlike the conventional GenSM and GenQSM that map data bits separately to the signal and spatial constellation points, we map $n$ data bits jointly to a transmit vector $\hat{\mathbf{x}} \in \mathbb{C}^{N_{t}}$, which can be viewed as a high-dimensional symbol. In such a system, the number of used TACs does not need to be a power of two, and the signal constellations used for different TACs are not required to be the same [15].

With $\hat{\mathbf{x}}$ being transmitted, the receive signal vector $\hat{\mathbf{y}} \in \mathbb{C}^{N_{r}}$ can be written as

$$
\hat{\mathbf{y}}=\sqrt{\rho} \hat{\mathbf{H}} \hat{\mathbf{x}}+\hat{\mathbf{n}},
$$

where $\rho$ denotes the average receive SNR; $\hat{\mathbf{H}} \in \mathbb{C}^{N_{r} \times N_{t}}$ is the channel matrix and $\hat{\mathbf{n}} \in \mathbb{C}^{N_{r}}$ represents the complex Gaussian noise vector with zero mean and unit variance, i.e., $\hat{\mathbf{n}} \sim \mathcal{C N}\left(\mathbf{0}, \mathbf{I}_{N_{r}}\right)$. The transmission can be re-expressed in the real domain as

$$
\mathbf{y}=\sqrt{\rho} \mathbf{H x}+\mathbf{n},
$$

where

$$
\begin{aligned}
& \mathbf{y}=\left[\begin{array}{c}
\Re(\hat{\mathbf{y}}) \\
\Im(\hat{\mathbf{y}})
\end{array}\right], \quad \mathbf{H}=\left[\begin{array}{cc}
\Re(\hat{\mathbf{H}}) & -\Im(\hat{\mathbf{H}}) \\
\Im(\hat{\mathbf{H}}) & \Re(\hat{\mathbf{H}})
\end{array}\right], \\
& \mathbf{x}=\left[\begin{array}{r}
\Re(\hat{\mathbf{x}}) \\
\Im(\hat{\mathbf{x}})
\end{array}\right], \quad \mathbf{n}=\left[\begin{array}{c}
\Re(\hat{\mathbf{n}}) \\
\Im(\hat{\mathbf{n}})
\end{array}\right]
\end{aligned}
$$

We use $\mathcal{X}_{N}=\left\{\mathbf{x}_{1}, \mathbf{x}_{2}, \cdots, \mathbf{x}_{N}\right\}$ of size $N=2^{n}$ to represent the transmit vector set, where $\mathbf{x}_{i}=\left[\Re\left(\hat{\mathbf{x}}_{i}\right)^{T}, \Im\left(\hat{\mathbf{x}}_{i}\right)^{T}\right]^{T} \in$ $\mathbb{R}^{2 N_{t}}$. It is assumed that the transmit vectors in $\mathcal{X}_{N}$ are under a unit average power constraint, which is expressed as

$$
P\left(\mathcal{X}_{N}\right)=\mathbb{E}\left(\|\mathbf{x}\|^{2}\right)=\frac{1}{N} \sum_{i=1}^{N} \mathbf{x}_{i}{ }^{T} \mathbf{x}_{i} \leq 1 .
$$

Besides, they are also under a sparsity constraint, because the number of data streams sent via RF chains should be less than or equal to the number of RF chains. Specifically, for GenSM, the sparsity constraint is given by

$$
\left\|\mathbf{x}_{i}^{R}+j \mathbf{x}_{i}^{I}\right\|_{0} \leq N_{R F}, i=1,2, \cdots, N, j=\sqrt{-1},
$$

while for GenQSM, the sparsity constraint is given by

$$
\left\|\mathbf{x}_{i}^{R}\right\|_{0} \leq N_{R F},\left\|\mathbf{x}_{i}^{I}\right\|_{0} \leq N_{R F}, i=1,2, \cdots, N .
$$

where $\mathbf{x}_{i}^{R} \triangleq\left[\mathbf{x}_{i}(1), \mathbf{x}_{i}(2), \cdots, \mathbf{x}_{i}\left(N_{t}\right)\right]^{T}=\Re(\hat{\mathbf{x}}) \in \mathbb{R}^{N_{t}}$ and $\mathbf{x}_{i}^{I} \triangleq\left[\mathbf{x}_{i}\left(N_{t}+1\right), \mathbf{x}_{i}\left(N_{t}+2\right), \cdots, \mathbf{x}_{i}\left(2 N_{t}\right)\right]^{T}=\Im(\hat{\mathbf{x}}) \in \mathbb{R}^{N_{t}}$ represent the vectors being composed of the first $N_{t}$ entries and the last $N_{t}$ entries of vector $\mathbf{x}_{i}$, respectively.

Remark: The sparsity constraints for the transmit vectors of GenSM and GenQSM are different from their conventional counterparts, because the positions of non-zero elements in the transmit vectors are constrained. That is, let $\mathcal{I}_{i}^{R}$ and $\mathcal{I}_{i}^{I}$ be the index sets of non-zero elements in $\mathbf{x}_{i}^{R}$ and $\mathbf{x}_{i}^{I}$, respectively. For GenSM, the sparsity constraint can be re-expressed as

$$
\left|\mathcal{I}_{i}^{I} \cup \mathcal{I}_{i}^{R}\right| \leq N_{R F}, i=1,2, \cdots, N .
$$

For GenQSM, it is

$$
\left|\mathcal{I}_{i}^{I}\right| \leq N_{R F},\left|\mathcal{I}_{i}^{R}\right| \leq N_{R F}, i=1,2, \cdots, N .
$$


For comparison purposes, we also give the conventional sparsity constraint without the position limitation as follows:

$$
\left\|\mathbf{x}_{i}\right\|_{0} \leq 2 N_{R F}, i=1,2, \cdots, N
$$

or

$$
\left|\mathcal{I}_{i}^{I}\right|+\left|\mathcal{I}_{i}^{R}\right| \leq 2 N_{R F}, \quad i=1,2, \cdots, N
$$

\section{B. Channel Model}

In this paper, a specific transmit-correlated Rayleigh channel model is adopted and its channel matrix can be written as [16]

$$
\hat{\mathbf{H}}=\hat{\mathbf{H}}_{w} \mathbf{R}_{t x}^{1 / 2}
$$

where $\hat{\mathbf{H}}_{w} \in \mathbb{C}^{N_{r} \times N_{t}}$ represents a complex Gaussian matrix with $\left[\hat{\mathbf{H}}_{w}\right]_{k, l} \sim \mathcal{C N}(0,1)$ and $\mathbf{R}_{t x} \in \mathbb{C}^{N_{t} \times N_{t}}$ denotes the transmit correlation matrix. The correlation weight matrix $\mathbf{R} \in$ $\mathbb{R}^{2 N_{t} \times 2 N_{t}}$ can be written in the real domain as

$$
\mathbf{R}=\left[\begin{array}{cc}
\Re\left(\mathbf{R}_{t x}^{1 / 2}\right) & -\Im\left(\mathbf{R}_{t x}^{1 / 2}\right) \\
\Im\left(\mathbf{R}_{t x}^{1 / 2}\right) & \Re\left(\mathbf{R}_{t x}^{1 / 2}\right)
\end{array}\right] .
$$

Despite the specific channel model is used as an example for illustration purposes, it should be noted that the proposed optimization strategies and obtained results are applicable to generalized channel models.

\section{UNIFIED OPTIMIZATION PROBLEM FORMULATION BASED ON THE MMED CRITERION}

In this section, we formulate a unified optimization problem of the signal shaping for multiple-data-stream GenSM and GenQSM based on the MMED criterion in three cases that are without CSIT, and with statistical as well as instantaneous CSIT, respectively. Without CSIT, $\mathcal{X}_{N}$ is designed for maximizing the minimum Euclidean distance of the transmit vectors by

$$
\max d_{\min }\left(\mathcal{X}_{N}, \mathbf{I}_{2 N_{t}}\right)=\max \min _{\mathbf{x}_{i} \neq \mathbf{x}_{i^{\prime}} \in \mathcal{X}_{N}}\left\|\mathbf{x}_{i}-\mathbf{x}_{i^{\prime}}\right\|_{2} .
$$

With statistical CSIT (i.e., R), $\mathcal{X}_{N}$ is designed for maximizing the minimum Euclidean distance of the correlation wighted transmit vectors by

$$
\max d_{\min }\left(\mathcal{X}_{N}, \mathbf{R}\right)=\max \min _{\mathbf{x}_{i} \neq \mathbf{x}_{i^{\prime}} \in \mathcal{X}_{N}}\left\|\mathbf{R}\left(\mathbf{x}_{i}-\mathbf{x}_{i^{\prime}}\right)\right\|_{2} .
$$

With perfect CSIT, $\mathcal{X}_{N}$ is designed for maximizing the minimum Euclidean distance of noise-free receive signal vectors by

$$
\max d_{\min }\left(\mathcal{X}_{N}, \mathbf{H}\right)=\max \min _{\mathbf{x}_{i} \neq \mathbf{x}_{i^{\prime}} \in \mathcal{X}_{N}}\left\|\mathbf{H}\left(\mathbf{x}_{i}-\mathbf{x}_{i^{\prime}}\right)\right\|_{2} .
$$

According to the optimization problems formulated above, we use a $2 N_{t}$-column weight matrix A to represent $\mathbf{I}_{2 N_{t}}, \mathbf{R}$ or $\mathbf{H}$, and rewrite three objective functions in a unified form to be

$$
\max d_{\min }\left(\mathcal{X}_{N}, \mathbf{A}\right)=\max \min _{\mathbf{x}_{i} \neq \mathbf{x}_{i^{\prime}} \in \mathcal{X}_{N}}\left\|\mathbf{A}\left(\mathbf{x}_{i}-\mathbf{x}_{i^{\prime}}\right)\right\|_{2}
$$

Therefore, the signal shaping optimization problems for GenS$\mathrm{M}$ and GenQSM of the aforementioned three cases can be formulated as

$$
\begin{aligned}
\text { P1) : } & \text { Given }: \\
\text { Find }: & \mathbf{A}, N_{R F}, N=\left\{\mathbf{x}_{1}, \mathbf{x}_{2}, \cdots, \mathbf{x}_{N}\right\} \\
\text { Maximize }: & d_{\min }\left(\mathcal{X}_{N}, \mathbf{A}\right) \\
\text { Subject to }: & \left|\mathcal{X}_{N}\right|=N \\
& P\left(\mathcal{X}_{N}\right) \leq 1 \\
& \left\|\mathbf{x}_{i}^{R}+j \mathbf{x}_{i}^{I}\right\|_{0} \leq N_{R F} \\
& i=1,2, \cdots, N,
\end{aligned}
$$

and

$$
\begin{aligned}
(\mathbf{P 2}): \quad \text { Given }: & \mathbf{A}, N_{R F}, N \\
\text { Find : } & \mathcal{X}_{N}=\left\{\mathbf{x}_{1}, \mathbf{x}_{2}, \cdots, \mathbf{x}_{N}\right\} \\
\text { Maximize }: & d_{\min }\left(\mathcal{X}_{N}, \mathbf{A}\right) \\
\text { Subject to : } & \left|\mathcal{X}_{N}\right|=N \\
& P\left(\mathcal{X}_{N}\right) \leq 1 \\
& \left\|\mathbf{x}_{i}^{R}\right\|_{0} \leq N_{R F},\left\|\mathbf{x}_{i}^{I}\right\|_{0} \leq N_{R F} \\
& i=1,2, \cdots, N,
\end{aligned}
$$

respectively. In (P1) and (P2), (17a), (18a) represent the size constraints; (17b), (18b) are the unit average power constraints and $(17 \mathrm{c}),(18 \mathrm{c})$ are the special sparsity constraints.

To replace the sparsity constraints in (17c) and (18c), we express $\mathbf{x}_{i}$ as $\mathbf{x}_{i}=\mathbf{F}_{k} \mathbf{s}_{l}^{k}$, where $\mathbf{F}_{k} \in \mathbb{R}^{2 N_{t} \times 2 N_{R F}}$ is a matrix that corresponds to the $k$ th TAC and $\mathbf{s}_{l}^{k} \in \mathbb{R}^{2 N_{R F}}$ is the $l$ th data symbol vector when $\mathbf{F}_{k}$ is activated. For GenSM, $\mathbf{F}_{k}$ can be expressed as

$$
\mathbf{F}_{k}^{\mathrm{GenSM}}=\left[\begin{array}{cc}
\mathbf{C}_{u} & \mathbf{0} \\
\mathbf{0} & \mathbf{C}_{u}
\end{array}\right],
$$

where $\mathbf{C}_{u} \in \mathbb{R}^{N_{t} \times N_{R F}}$ is an antenna selection matrix composed of $N_{R F}$ basis vectors of dimension $N_{t}$. Let $\mathcal{F}_{\text {GenSM }}$ denote the set of all feasible $\mathbf{F}_{k}^{\mathrm{GenSM}}$. Since there are totally $\left(\begin{array}{c}N_{t} \\ N_{R F}\end{array}\right)$ different $\mathbf{C}_{u}$, the number of feasible $\mathbf{F}_{k}^{\mathrm{GenSM}}$ in $\mathcal{F}_{\text {GenSM }}$ is $\left(\begin{array}{c}N_{t} \\ N_{R F}\end{array}\right)$, corresponding to $\left(\begin{array}{c}N_{t} \\ N_{R F}\end{array}\right)$ feasible TACs. For GenQSM, $\mathbf{F}_{k}$ can be given by

$$
\mathbf{F}_{k}^{\mathrm{GenQSM}}=\left[\begin{array}{cc}
\mathbf{C}_{u} & \mathbf{0} \\
\mathbf{0} & \mathbf{C}_{v}
\end{array}\right],
$$

where $\mathbf{C}_{u}, \mathbf{C}_{v} \in \mathbb{R}^{N_{t} \times N_{R F}}$ are two independent antenna selection matrices. Let $\mathcal{F}_{\text {GenQSM }}$ denote the set of all feasible $\mathbf{F}_{k}^{\text {GenQSM }}$. As there are $\left(\begin{array}{c}N_{t} \\ N_{R F}\end{array}\right)$ different $\mathbf{C}_{u}$ and $\mathbf{C}_{v}$, the number of feasible $\mathbf{F}_{k}^{\text {GenQSM }}$ in $\mathcal{F}_{\text {GenQSM }}$ is $\left(\begin{array}{c}N_{t} \\ N_{R F}\end{array}\right)^{2}$, corresponding to $\left(\begin{array}{c}N_{t} \\ N_{R F}\end{array}\right)^{2}$ feasible TACs.

For unification, we use $\mathbf{F}_{k}$ to represent $\mathbf{F}_{k}^{\mathrm{GenSM}}$ or $\mathbf{F}_{k}^{\mathrm{GenQSM}}$ and $\mathcal{F}$ to represent $\mathcal{F}_{\text {GenSM }}$ or $\mathcal{F}_{\text {GenQSM }}$. The $k$ th signal constellation $\mathcal{S}_{k}$ is defined as the set of $\mathbf{s}_{l}^{k}$ when $\mathbf{F}_{k}$ is activated and the set of all signal constellations is represented 
by $\mathcal{Z}=\left\{\mathcal{S}_{1}, \mathcal{S}_{2}, \cdots, \mathcal{S}_{|\mathcal{F}|}\right\}$. Based on these denotations, $(\mathbf{P 1})$ and $(\mathbf{P 2})$ can be expressed in a unified manner as follows:

$$
\begin{aligned}
& \text { (OP) : } \quad \text { Given : } \mathbf{A}, N, \mathcal{F} \\
& \text { Find : } \mathcal{Z}=\left\{\mathcal{S}_{1}, \mathcal{S}_{2}, \cdots, \mathcal{S}_{|\mathcal{F}|}\right\} \\
& \text { Maximize : } d_{\min }(\mathcal{F}, \mathcal{Z}, \mathbf{A}) \\
& \text { Subject to : } \sum_{k=1}^{|\mathcal{F}|}\left|\mathcal{S}_{k}\right|=N \\
& P(\mathcal{Z}) \leq 1,
\end{aligned}
$$

where

$$
d_{\min }(\mathcal{F}, \mathcal{Z}, \mathbf{A})=\min _{\substack{\mathbf{F}_{k} \mathbf{s}_{l}^{k} \neq \mathbf{F}_{k^{\prime}} \mathbf{s}_{l^{\prime}}^{k^{\prime}} \\ \mathbf{F}_{l}, \mathbf{F}_{k^{\prime}} \in \mathcal{F} \\ \mathrm{s}_{l}^{k} \in \mathcal{S}_{k}, s^{\prime} \in \mathcal{S}_{k^{\prime}} \\ \mathcal{S}_{k}, \mathcal{S}_{k^{\prime}} \in \mathcal{Z}}}\left\|\mathbf{A}\left(\mathbf{F}_{k} \mathbf{s}_{l}^{k}-\mathbf{F}_{k^{\prime}} \mathbf{s}_{l^{\prime}}^{k^{\prime}}\right)\right\|_{2},
$$

and

$$
P(\mathcal{Z})=\frac{1}{N} \sum_{k=1}^{|\mathcal{Z}|} \sum_{l=1}^{\left|\mathcal{S}_{k}\right|}\left(\mathbf{s}_{l}^{k}\right)^{T} \mathbf{s}_{l}^{k} .
$$

\section{Optimization-Based Signal Shaping}

Since the problem (OP) for optimizing $\mathcal{X}_{N}$ has been reformulated to search the optimal signal constellations $\mathcal{S}_{1}$, $\mathcal{S}_{2}, \ldots, \mathcal{S}_{|\mathcal{F}|}$ in the last section, the problem becomes a set optimization problem including the set size optimization and the set entry optimization. We analyze both sub-problems with details in the following subsections.

\section{A. Set Size Optimization}

The set size optimization is a non-negative integer program$\operatorname{ming}$ satisfying $\sum_{k=1}^{|\mathcal{F}|}\left|\mathcal{S}_{k}\right|=N$. There are a total number of $\left(\begin{array}{c}N+|\mathcal{F}|-1 \\ |\mathcal{F}|-1\end{array}\right)$ feasible solutions [39], which is rather large. Taking a MIMO system with $N_{t}=4, N_{R F}=2$ and $N=16$ (i.e., $n=4$ bpcu) as an example, $\left|\mathcal{F}_{\text {GenSM }}\right|=\left(\begin{array}{l}4 \\ 2\end{array}\right)=6$ and $\left|\mathcal{F}_{\text {GenQSM }}\right|=\left(\begin{array}{l}4 \\ 2\end{array}\right)^{2}=36$. We can easily calculate that there are $\left(\begin{array}{c}16+6-1 \\ 6-1\end{array}\right) \approx 2 \times 10^{4}$ feasible size solutions for GenSM and $\left(\begin{array}{c}16+36-1 \\ 36-1\end{array}\right) \approx 7.2 \times 10^{12}$ solutions for GenQSM, respectively. Therefore, exhaustive search for the optimal solution is infeasible, because the set entry optimization is needed for each feasible set size solution. In [16] that considers single-datastream transmission without CSIT, a greedy recursive method was proposed by finding the optimal set size solution for $\mathcal{X}_{N}$ with size $N$ according to $\mathcal{X}_{N-1}$ with size $N-1$. The recursive method shows comparable performance to the exhaustive search in the single-data-steam cases but demands much lower complexity [16]. Thus, we also suggest the use of its extension in multiple-data-steam cases. To introduce the extension, we define a constellation partition matrix $\mathbf{W}_{N} \in \mathbb{R}^{2 N_{t} \times 2 N N_{R F}}$ for $\mathcal{X}_{N}$ with size $N$ as

$\mathbf{W}_{N} \triangleq[\overbrace{\mathbf{F}_{1}, \cdots, \mathbf{F}_{1}}^{\left|\mathcal{S}_{1}\right|} \overbrace{\mathbf{F}_{2}, \cdots, \mathbf{F}_{2}}^{\left|\mathcal{S}_{2}\right|}, \cdots, \cdots, \overbrace{\mathbf{F}_{|\mathcal{F}|}, \cdots, \mathbf{F}_{|\mathcal{F}|}}^{\left|\mathcal{S}_{|\mathcal{F}|}\right|}]_{(24)}$, which can be regarded as an indicator of the signal constellation sizes $\left\{\left|\mathcal{S}_{k}\right|\right\}$ and the number of $\mathbf{F}_{k}$ in $\mathbf{W}_{N}$ indicates the signal constellation size $\left|\mathcal{S}_{k}\right|$ for the $k$ th TAC. With the definition of $\mathbf{W}_{N}$, the extension of the recursive design in multiple-data-stream cases can be described as follows. Given $\mathbf{W}_{N-1}$, we can choose an $\mathbf{F}_{k} \in \mathcal{F}$ to adjoin $\mathbf{W}_{N-1}$ generating $|\mathcal{F}|$ candidates of $\mathbf{W}_{N}$. For each candidate of $\mathbf{W}_{N}$, we perform set size optimization and obtain the corresponding candidates of $\mathcal{X}_{N}$. Then, by comparing all the candidates of $\mathcal{X}_{N}$, we can obtain a suboptimal $\mathcal{X}_{N}$ among all the candidates and the corresponding suboptimal $\mathbf{W}_{N}$. Based on this principle, we use the optimal $\mathcal{X}_{2}$ and $\mathbf{W}_{2}$, which can be obtained by exhaustive search, to find a suboptimal $\mathcal{X}_{3}$ and $\mathbf{W}_{3}$, then $\mathcal{X}_{4}$ and $\mathbf{W}_{4}$ and so on until the size constraint is satisfied.

\section{B. Set Entry Optimization}

Given a fixed $\mathbf{W}_{N}$, the sizes of $\mathcal{S}_{1}, \mathcal{S}_{2}, \cdots, \mathcal{S}_{|\mathcal{F}|}$ are determined and we now need to optimize the set entries in each set to maximize $d_{\min }(\mathcal{F}, \mathcal{Z}, \mathbf{A})$ in $(\mathbf{O P})$. To solve the problem, we define $\mathbf{S}_{l}^{k} \triangleq \operatorname{diag}\left(\mathbf{s}_{l}^{k}\right)$ for all $k=1,2, \cdots,|\mathcal{F}|$, $l=1,2, \cdots,\left|\mathcal{S}_{k}\right|$, and a diagonal matrix $\mathbf{D}_{\mathbf{q}}$ of dimension $2 N N_{R F} \times 2 N N_{R F}$ as

$$
\mathbf{D}_{\mathbf{q}} \triangleq\left[\begin{array}{ccccccc}
\mathbf{S}_{1}^{1} & \mathbf{0} & \mathbf{0} & \cdots & \mathbf{0} & \mathbf{0} & \mathbf{0} \\
\mathbf{0} & \ddots & \mathbf{0} & \cdots & . & \mathbf{0} & \mathbf{0} \\
\mathbf{0} & \mathbf{0} & \mathbf{S}_{\left|\mathcal{S}_{1}\right|}^{1} & \cdots & \mathbf{0} & . & \mathbf{0} \\
\vdots & \vdots & \vdots & \ddots & \vdots & \vdots & \vdots \\
\mathbf{0} & \cdot & \mathbf{0} & \cdots & \mathbf{S}_{1}^{|\mathcal{F}|} & \mathbf{0} & \mathbf{0} \\
\mathbf{0} & \mathbf{0} & . & \cdots & \mathbf{0} & \ddots & \mathbf{0} \\
\mathbf{0} & \mathbf{0} & \mathbf{0} & \cdots & \mathbf{0} & \mathbf{0} & \mathbf{S}_{\left|\mathcal{S}_{|\mathcal{F}|}\right|}^{|\mathcal{F}|}
\end{array}\right],
$$

as well as a vector $\mathbf{e}_{i} \in \mathbb{R}^{2 N N_{R F}}$ as $\mathbf{e}_{i} \triangleq \mathbf{g}_{i} \otimes \mathbf{1}_{2 N_{R F}}$ where $\mathbf{g}_{i}$ is the $i$ th $N$-dimensional vector basis with all zeros except the $i$ th entry being one. Based on these definitions, the square of the pairwise Euclidean distances can be expressed as

$$
\begin{aligned}
\left\|\mathbf{A} \mathbf{x}_{i}-\mathbf{A} \mathbf{x}_{i^{\prime}}\right\|_{2}^{2} & =\left\|\mathbf{A} \mathbf{W}_{N} \mathbf{D}_{\mathbf{q}} \mathbf{e}_{i}-\mathbf{A} \mathbf{W}_{N} \mathbf{D}_{\mathbf{q}} \mathbf{e}_{i^{\prime}}\right\|_{2}^{2} \\
& =\left(\mathbf{e}_{i}-\mathbf{e}_{i^{\prime}}\right)^{T} \mathbf{D}_{\mathbf{q}}^{T} \mathbf{W}_{N}^{T} \mathbf{A}^{T} \mathbf{A} \mathbf{W}_{N} \mathbf{D}_{\mathbf{q}}\left(\mathbf{e}_{i}-\mathbf{e}_{i^{\prime}}\right) \\
& =\operatorname{Tr}\left(\mathbf{D}_{\mathbf{q}}^{T} \mathbf{R}_{\mathbf{A W}} \mathbf{D}_{\mathbf{q}} \Delta \mathbf{E}_{i i^{\prime}}\right),
\end{aligned}
$$

where $\mathbf{R}_{\mathbf{A W}}=\mathbf{W}_{N}^{T} \mathbf{A}^{T} \mathbf{A} \mathbf{W}_{N}$ and $\Delta \mathbf{E}_{i i^{\prime}}=\left(\mathbf{e}_{i}-\mathbf{e}_{i^{\prime}}\right)\left(\mathbf{e}_{i}-\mathbf{e}_{i^{\prime}}\right)^{T}$. Adopting the equality $\operatorname{Tr}\left(\mathbf{D}_{\mathbf{u}} \mathbf{U} \mathbf{D}_{\mathbf{v}} \mathbf{V}^{T}\right)=\mathbf{u}^{T}(\mathbf{U} \odot \mathbf{V}) \mathbf{v}$, where $\mathbf{D}_{\mathbf{u}}=\operatorname{diag}(\mathbf{u})$ and $\mathbf{D}_{\mathbf{v}}=\operatorname{diag}(\mathbf{v})$, we rewrite (26) as

$$
\left\|\mathbf{A} \mathbf{x}_{i}-\mathbf{A} \mathbf{x}_{i^{\prime}}\right\|_{2}^{2}=\mathbf{q}^{T} \mathbf{Q}_{i i^{\prime}} \mathbf{q},
$$

where $\mathbf{q}=\operatorname{diag}\left\{\mathbf{D}_{\mathbf{q}}\right\} \in \mathbb{R}^{2 N N_{R F}}$ and $\mathbf{Q}_{i i^{\prime}}=\mathbf{R}_{\mathbf{A W}} \odot \Delta \mathbf{E}_{i i^{\prime}}^{T} \in$ $\mathbb{R}^{2 N N_{R F} \times 2 N N_{R F}}$. As a consequence, the unit power constraint can be expressed as

$$
P(\mathcal{Z})=\frac{1}{N} \operatorname{Tr}\left(\mathbf{D}_{\mathbf{q}} \mathbf{D}_{\mathbf{q}}^{T}\right)=\frac{1}{N} \mathbf{q}^{T} \mathbf{q} \leq 1 .
$$


Based on the above reformulations, the set entry optimization becomes

$$
\begin{aligned}
&(\mathbf{S}-\mathbf{O P}): \quad \text { Given }: \mathbf{Q}_{i i^{\prime}}, \forall i \neq i^{\prime} \in\{1,2, \cdots, N\} \\
& \text { Find }: \mathbf{q} \\
& \text { Maximize }: \min \mathbf{q}^{T} \mathbf{Q}_{i j} \mathbf{q} \\
& \text { Subject to }: \mathbf{q}^{T} \mathbf{q} \leq N
\end{aligned}
$$

By introducing an auxiliary variable $\tau$, the optimization problem can be equivalently transformed to be

$$
\begin{aligned}
(\mathbf{S}-\mathbf{O P}-\mathbf{a}): \text { Given }: & \mathbf{Q}_{i i^{\prime}}, \forall i \neq i^{\prime} \in\{1,2, \cdots, N\} \\
\text { Find }: & \mathbf{q}, \tau \\
\text { Maximize }: & \tau \\
\text { Subject to }: & \mathbf{q}^{T} \mathbf{Q}_{i j} \mathbf{q} \geq \tau, \forall i \neq i^{\prime} \in\{1,2, \cdots, N\} \\
& \mathbf{q}^{T} \mathbf{q} \leq N
\end{aligned}
$$

Problem (S-OP-a) is a non-convex large-scale QCQP problem with $2 N N_{R F}$ variables and $\left(\begin{array}{c}N \\ 2\end{array}\right)$ constraints, and can be solved by the iterative algorithm developed in [30] with complexity about $\mathcal{O}\left(N^{4} N_{R F}^{2}\right)$ in each iteration ${ }^{1}$. Moreover, because the minimum Euclidean distance is monotonically increasing with the increase of the average power, problem (S-OP-a) that maximizes the minimum distance subject to an average power constraint can be reformulated to an optimization problem minimizing the average power for a target minimum distance, which can be expressed as

$$
\begin{aligned}
&(\mathbf{S - O P}-\mathbf{b}): \text { Given }: \mathbf{Q}_{i i^{\prime}}, \forall i \neq i^{\prime} \in\{1,2, \cdots, N\} \\
& \text { Find }: \mathbf{q} \\
& \text { Minimize }: \mathbf{q}^{T} \mathbf{q} \\
& \text { Subject to }: \mathbf{q}^{T} \mathbf{Q}_{i j} \mathbf{q} \geq d, \forall i \neq i^{\prime} \in\{1,2, \cdots, N\},
\end{aligned}
$$

where $d$ is the target minimum distance. The problem (S-OPb) can be solved by the Lagrangian method developed in [38]. The complexity is also around $\mathcal{O}\left(N^{4} N_{R F}^{2}\right)$ in each iteration ${ }^{1}$, but fortunately the algorithm in [38] converges faster than that in [30]. Additionally, it should be noted that problem (S-OPb) is formulated without any power constraint and one should further scale the optimized transmit vectors to meet the unit average power constraint.

In the paper, we use the advanced algorithm developed in [38] to solve the formulated QCQP problem (S-OP-b). The number of iterations that the algorithm in [38] takes to converge is one of the key factors dominating the computational complexity. Furthermore, we demonstrate how the parameters $N$ and $N_{R F}$ affect the key factor in Fig. 2. In the simulations with $N_{t}=4$ and $N_{r}=4$, the number of iterations that the algorithm in [38] takes to converge is obtained by averaging over 100 realizations. Observing the results demonstrated in Fig. 2, we find that the average number of iterations that the algorithm [38] takes to converge for solving (S-OP-b) slightly increases as $N_{R F}$ and $N$ increase, because both of them

\footnotetext{
${ }^{1}$ We omit the other terms in the complexity analysis in [30] and [38] for simplicity, since $N$ is much larger than other components constituting the complexity.
}

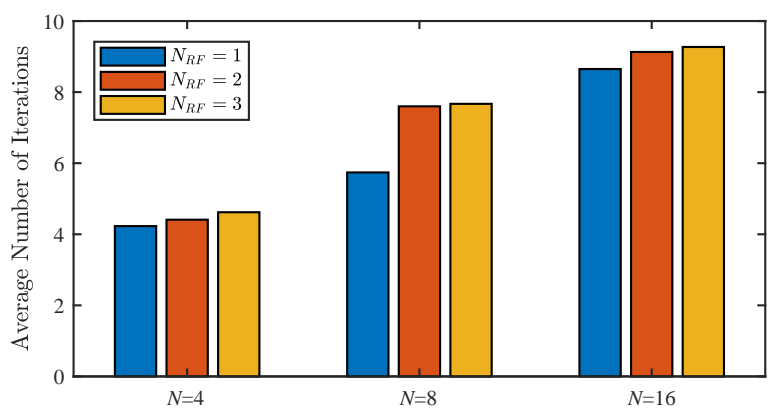

Fig. 2. Average number of iterations that the algorithm proposed in [38] takes to converge for solving (S-OP-b).

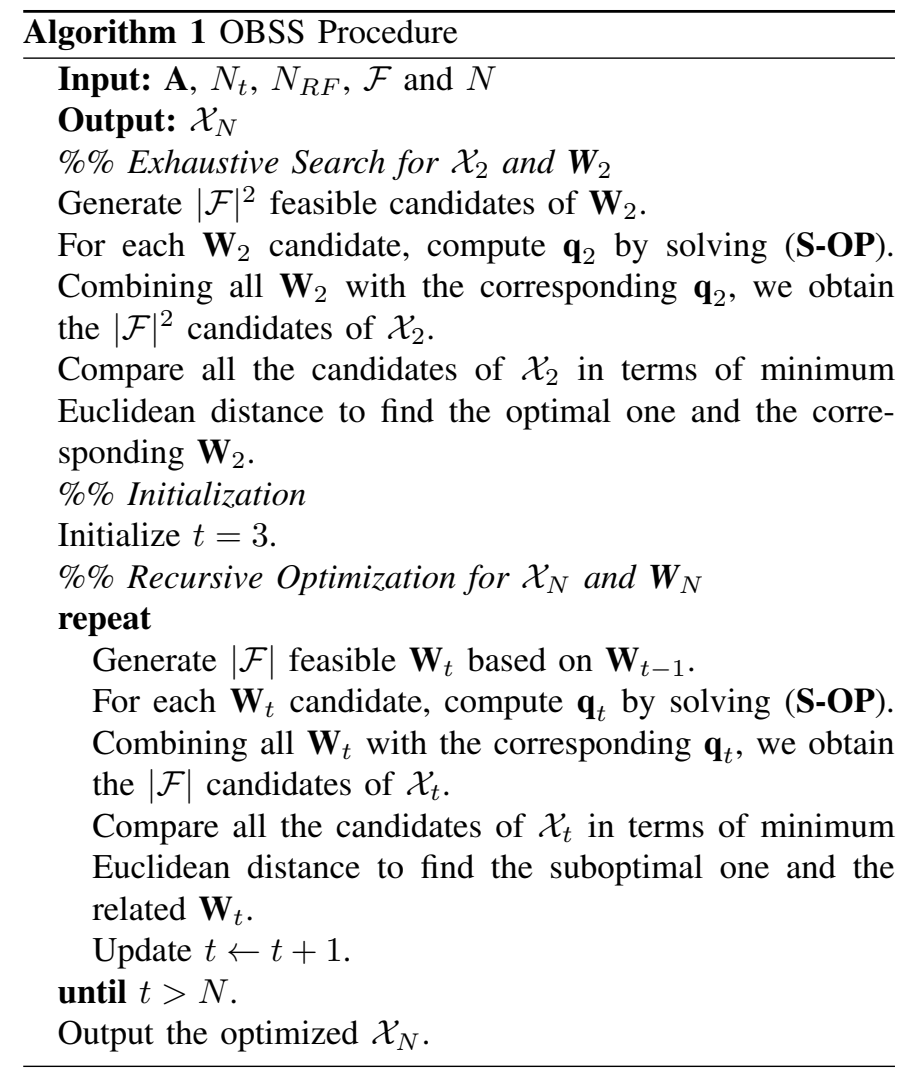

determine the number of variables and $N$ solely determines the number of constraints.

\section{Complexity Analysis}

For clearly viewing the detailed design procedure of OBSS, we list it in Algorithm 1, where $\mathbf{q}_{t}, \mathbf{W}_{t}$ and $\mathcal{X}_{t}$ are the temporary variables in the $t$ th iteration. The complexity of Algorithm 1 is determined by the exhaustive search for $\mathcal{X}_{2}$ and the recursive optimization for $\mathcal{X}_{N}$. In the exhaustive search for $\mathcal{X}_{2}$, we need to solve (S-OP) for $|\mathcal{F}|^{2}$ times. The aggregate complexity is $\mathcal{O}\left(|\mathcal{F}|^{2} N_{\text {iter }_{2}} 2^{4} N_{R F}^{2}\right)$, where $N_{\text {iter }_{2}}$ denotes the number of iterations for solving (S-OP). It is obvious that the complexity is rather low and thereby negligible. In the recursive optimization for $\mathcal{X}_{N}$, we need to solve (S-OP) for 
TABLE I

COMPARISONS AMONG DifFERENT Signal SHAPING APPROACHES

\begin{tabular}{|c|c|c|c|c|}
\hline Approaches & Number of variables & Number of constraints & Complexity & Applications \\
\hline OBSS & $2 N N_{R F}$ & $\left(\begin{array}{c}N \\
2\end{array}\right)$ & $\mathcal{O}\left(N_{\text {iter }}|\mathcal{F}| N^{5} N_{R F}^{2}\right)$ & GenSM/GenQSM \\
\hline Diagonal precoding [38] & $2\left|\mathcal{F}_{s}\right| N_{R F}$ & $\left(\begin{array}{c}N \\
2\end{array}\right)$ & $\mathcal{O}\left(N_{\text {iter }}^{D}\left|\mathcal{F}_{s}\right|^{2} N_{R F}^{2} N^{2}\right)$ & GenSM \\
\hline Full precoding [38] & $2\left|\mathcal{F}_{s}\right| N_{R F}^{2}$ & $\left(\begin{array}{c}N \\
2\end{array}\right)$ & $\mathcal{O}\left(N_{\text {iter }}^{F}\left|\mathcal{F}_{s}\right|^{2} N_{R F}^{4} N^{2}\right)$ & GenSM \\
\hline CBSS & - & - & $\mathcal{O}\left(N_{c}^{2}\right)$ & GenSM/GenQSM \\
\hline
\end{tabular}

$|\mathcal{F}|$ times for designing $\mathcal{X}_{t}$ and the aggregate complexity is

$$
\mathrm{C}=\sum_{t=3}^{N} \mathcal{O}\left(N_{\text {iter }_{t}}|\mathcal{F}| t^{4} N_{R F}^{2}\right) \approx \mathcal{O}\left(N_{\text {iter }_{t}}|\mathcal{F}| N^{5} N_{R F}^{2}\right),
$$

where $N_{\text {iter }_{t}}$ represents the number of iterations for solving (S-OP) to attain $\mathcal{X}_{t}$ and we assume $\left\{N_{\text {iter }_{t}}\right\}$ are of the same order for any $t$.

\section{Remarks}

The proposed OBSS approach aims to directly optimize the transmit vectors, which is different from the precoding-aided signal shaping proposed in [38] that modifies a given signal constellation of size $M^{N_{R F}}$ via a diagonal preceding matrix or a full precoding matrix for each TAC, where they assume $M$ ary modulation is adopted for each data stream transmission. Even though the diagonal precoder and the full precoder can be designed by solving a similar QCQP problem as (S-OP), there exists a difference in the number of optimization variables. For the diagonal precoder optimization, the total number of variables is $2\left|\mathcal{F}_{s}\right| N_{R F}$, while for the full precoder optimization, the total number of variables is $2\left|\mathcal{F}_{s}\right| N_{R F}^{2}$, where $\mathcal{F}_{s}$ is a selected subset of $\mathcal{F}$ and $\left|\mathcal{F}_{s}\right|=2^{\left\lfloor\log _{2}|\mathcal{F}|\right\rfloor}$. Compared to $2\left|\mathcal{F}_{s}\right| N_{R F}$ and $2\left|\mathcal{F}_{s}\right| N_{R F}^{2}$, the number of variables $2 N N_{R F}$ in (S-OP) is much larger, because $N=\left|\mathcal{F}_{s}\right| M^{N_{R F}}$. Additionally, the number of constraints in (S-OP) and that in the precodingaided signal shaping in [38] are the same. That is, the scale of the QCQP problem in the proposed OBSS approach is larger than those formulated in precoding-aided shaping methods proposed in [38]. As a result, the computational complexity of the proposed OBSS approach is much higher than the precoding-aided shaping. Anyway, the proposed OBSS approach can be treated as a generalized design and hence can yield better performance. Also, the global optimality of the proposed OBSS approach cannot be guaranteed, because of the greedy set size optimization and the non-convexity of (SOP) in the set entry optimization.

In summary, the OBSS approach is proposed for the sake of performance enhancement, whereas its complexity is rather high. It is affordable for off-line designs without CSIT or online designs with long-term invariant statistical CSIT. However, the complexity is a heavy burden for large-size signal shaping designs or adaptive designs with instantaneous CSIT. For adaptive designs, the complexity needs to be greatly reduced before implementing these designs in practice.

\section{Codebook-Based Signal Shaping}

To alleviate the heavy computation burden of the OBSS approach, we propose an alternative low-complexity CBSS

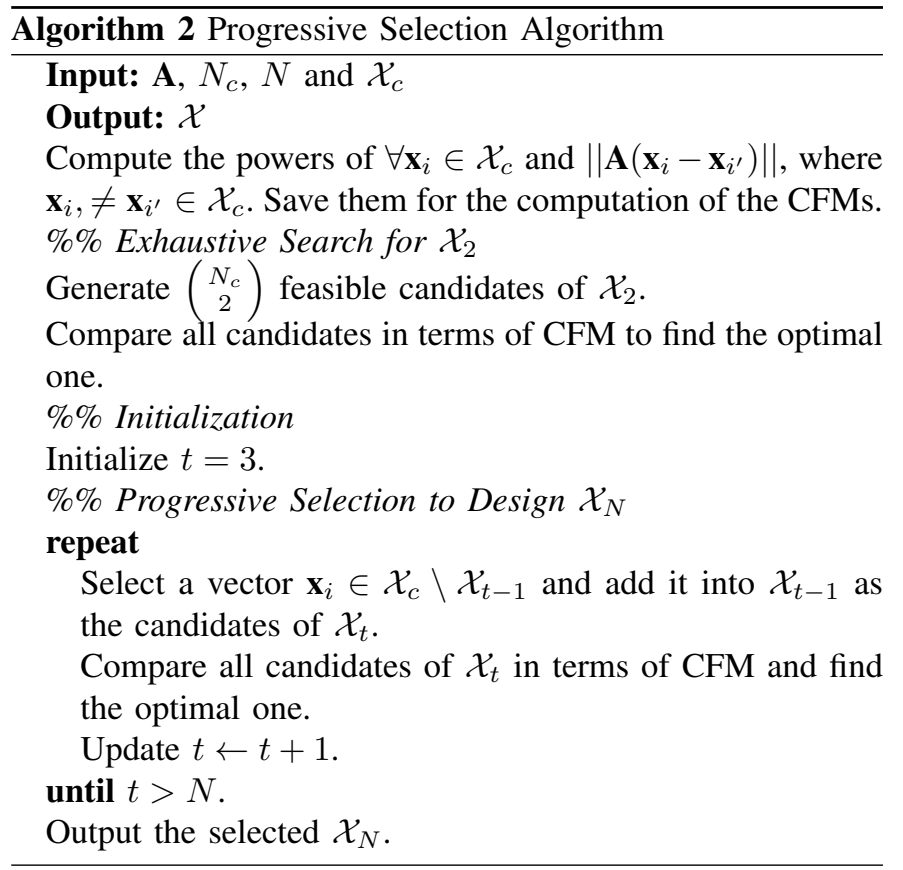

approach. We use a codebook generated by $M_{c}$-ary QAM modulation symbols, which is inspired by the work presented in [18]. With the codebook, there are $M_{c}^{N_{R F}}$ feasible signal constellation points and a total number of $N_{c}=|\mathcal{F}| M_{c}^{N_{R F}}$ feasible transmit vectors in the candidate set $\mathcal{X}_{c}$. Then, we need to select a subset of size $N$. To reduce the selection complexity, we progressively select vectors to maximize the constellation figure merit (CFM), which is equivalent to the MMED criterion under normalized power constraint and defined by [40]

$$
\operatorname{CFM}\left(\mathcal{X}_{N}\right) \triangleq \frac{d_{\min }\left(\mathcal{X}_{N}, \mathbf{A}\right)^{2}}{P\left(\mathcal{X}_{N}\right)} .
$$

This selection procedure is illustrated in Algorithm 2. The complexity of Algorithm 2 is dominated by the calculations of pairwise Euclidean distances, which is of the order $\mathcal{O}\left(N_{c}^{2}\right)$. For clarity, comprehensive comparisons among the proposed approach and other existing approaches are given in Table I. From Table I, we find that its complexity is much lower than existing approaches since it does not need to solve the large-scale QCQP problems, which enables its extensions to large-size signal shaping designs or adaptive designs with instantaneous CSIT.

\section{EXTENSIONS to MSER AND MMI DESIGNS}

In this section, we investigate the extensions to the signal shaping with the MSER and MMI as the design criteria, where 
perfect CSI is assumed to be available at the transmitter and the receiver.

\section{A. MSER Signal Shaping}

With a maximum likelihood (ML) detector employed at the receiver, the SER of GenSM/GenQSM systems is upper bounded by [24]

$$
\overline{P_{s}}\left(\mathcal{X}_{N}\right)=\frac{1}{2 N} \sum_{i=1}^{N} \sum_{\substack{i^{\prime}=1, i^{\prime} \neq i}}^{N} \exp \left(-\frac{\rho}{4}\left\|\mathbf{H}\left(\mathbf{x}_{i}-\mathbf{x}_{i^{\prime}}\right)\right\|_{2}^{2}\right) .
$$

Based on the re-formulation in Section IV-B with a fixed $\mathbf{W}_{N}$, we have

$$
\left\|\mathbf{H}\left(\mathbf{x}_{i}-\mathbf{x}_{i^{\prime}}\right)\right\|_{2}^{2}=\mathbf{q}^{T} \mathbf{Q}_{i i^{\prime}} \mathbf{q}
$$

and the upper bound can be re-expressed as

$$
\overline{P_{s}}(\mathbf{q})=\frac{1}{2 N} \sum_{i=1}^{N} \sum_{\substack{i^{\prime}=1, i^{\prime} \neq i}}^{N} \exp \left(-\frac{\rho}{4} \mathbf{q}^{T} \mathbf{Q}_{i i^{\prime}} \mathbf{q}\right) \text {. }
$$

Thus, the optimization problem to minimize the upper bound on SER can be formulated as

$$
\begin{aligned}
(\text { SER-OP }): \quad \text { Given }: & \mathbf{Q}_{i i^{\prime}}, \forall i \neq i^{\prime} \in\{1,2, \cdots, N\}, \rho \\
\text { Find }: & \mathbf{q} \\
\text { Minimize } & : \overline{P_{s}}(\mathbf{q}) \\
\text { Subject to } & : \mathbf{q}^{T} \mathbf{q} \leq N
\end{aligned}
$$

\section{B. MMI Signal Shaping}

According to the similar analysis in [26], the mutual information (MI) of GenSM/GenQSM systems given $\mathcal{X}$ as inputs can be expressed as

$\mathcal{I}(\mathbf{x} ; \mathbf{y} \mid \mathbf{H})=\log _{2} N-\cdots$

$\frac{1}{N} \sum_{i=1}^{N} \mathbb{E}_{\mathbf{n}}\left\{\log _{2} \sum_{i^{\prime}=1}^{N} \exp \left[-\rho\left(\left\|\mathbf{H}\left(\mathbf{x}_{i}-\mathbf{x}_{i^{\prime}}+\mathbf{n}^{2}\right)\right\|_{2}^{2}-\|\mathbf{n}\|_{2}^{2}\right)\right]\right\}$

The expression is lower bounded by [41]

$$
\begin{aligned}
\mathcal{I}_{L B}(\mathbf{x} ; \mathbf{y} \mid \mathbf{H})= & \log _{2} N+N_{r}\left(1-\log _{2} e\right) \\
& -\frac{1}{N} \sum_{i=1}^{N} \log _{2} \sum_{i^{\prime}=1}^{N} \exp \left(-\frac{\rho \| \mathbf{H}\left(\mathbf{x}_{i}-\mathbf{x}_{i^{\prime}} \|_{2}^{2}\right.}{2}\right) .
\end{aligned}
$$

Similarly, based on the expression $\left\|\mathbf{H}\left(\mathbf{x}_{i}-\mathbf{x}_{i^{\prime}}\right)\right\|_{2}^{2}=\mathbf{q}^{T} \mathbf{Q}_{i i^{\prime}} \mathbf{q}$ when $i \neq i^{\prime}$, we can obtain

$$
\begin{aligned}
\mathcal{I}_{L B}(\mathbf{q})= & \log _{2} N+N_{r}\left(1-\log _{2} e\right) \\
& -\frac{1}{N} \sum_{i=1}^{N} \log _{2}\left[1+\sum_{\substack{i^{\prime}=1 \\
i^{\prime} \neq i}}^{N} \exp \left(-\frac{\rho \mathbf{q}^{T} \mathbf{Q}_{i i^{\prime}} \mathbf{q}}{2}\right)\right] .
\end{aligned}
$$

Thus, the optimization problem to maximize the lower bound on MI can be formulated to be

$$
\begin{aligned}
(\text { MI-OP) }: \quad \text { Given }: & \mathbf{Q}_{i i^{\prime}}, \forall i \neq i^{\prime} \in\{1,2, \cdots, N\}, \rho \\
\text { Find }: & \mathbf{q} \\
\text { Maximize }: & \mathcal{I}_{L B}(\mathbf{q}) \\
\text { Subject to }: & \mathbf{q}^{T} \mathbf{q} \leq N
\end{aligned}
$$

\section{Remarks}

By replacing (S-OP-b) with (SEP-OP) and (MI-OP), the OBSS approach can be extended to the designs based on MSER and MMI criteria, respectively. This problems can also be solved by the existing algorithms, e.g., the algorithm proposed in [33] and the complexity are also of the same order, because the objective functions of (SEP-OP) and (MI-OP) are the functions of $\left\{\mathbf{q} \mathbf{Q}_{i i^{\prime}} \mathbf{q}\right\}$ and the computation of $\left\{\mathbf{q} \mathbf{Q}_{i i^{\prime}} \mathbf{q}\right\}$ dominates the computational complexity. Similarly, the CBSS approach can also be extended by using the SER and MI as selection metrics. We remark that the MSER and MMI metrics are reduced to the MMED metric in the high SNR regime, because the SER upper bound in (36) and the MI lower bound in (40) are dominated by the minimum Euclidean distance term with the help of the exponential operator. Moreover, it should be noted that compared to the MMED design, the MSER and MMI designs are SNR-dependent, which means that they need to be re-designed as SNR varies.

\section{Simulations AND DiscUSSIONS}

In the simulations, we investigate the performance of the proposed OBSS and CBSS approaches in variously configured $\left(N_{t}, N_{r}, N_{R F}, n\right)$ GenSM and GenQSM MIMO systems. The transmit-correlated Rayleigh channel model is adopted as described in Section II-B and the correlation matrix entries are defined by [16]

$$
\left[\mathbf{R}_{t x}\right]_{k, l}=\left\{\begin{array}{c}
\delta^{k-l}, \quad k \leq l \\
\left(\delta^{l-k}\right)^{\dagger}, \quad l>k
\end{array} k, l=1, \cdots, N_{t}\right.
$$

where $\delta$ represents the transmit correlation coefficient. Moreover, it is assumed that the transmitter and receiver both know $\delta$. We compare different signal shaping methods not only in the minimum Euclidean distance but also in the SER, where the ML detector is employed. Over transmit-correlated Rayleigh model, the asymptotic upper bound on the SER given $\mathcal{X}_{N}$ can be expressed as [42]

$$
\overline{P_{s 1}}=c \sum_{i=1}^{N} \sum_{i^{\prime}=1, i \neq i^{\prime}}^{N}\left\|\mathbf{R}\left(\mathbf{x}_{i}-\mathbf{x}_{i}^{\prime}\right)\right\|_{2}^{-2 N_{r}},
$$

where $c=\frac{\rho^{-N_{r}}}{N}\left(\begin{array}{c}2 N_{r}-1 \\ N_{r}\end{array}\right)$. With CSIT, the upper bound on the SER is given be

$$
\overline{P_{s 2}}=\mathbb{E}_{\mathbf{H}}\left[\overline{P_{s}}\left(\mathcal{X}_{N}\right)\right] .
$$

For clarity, we divide the section into three subsections. In the first subsection, we compare the proposed OBSS solution with the sole signal constellation optimized results in openloop systems without CSIT and closed-loop systems with statistical CSIT, where the optimal signal constellation has already been known and adopted for comparison. In the second subsection, we investigate the proposed OBSS and CBSS optimization strategies in closed-loop systems with instantaneous CSIT and compare them with the precoding-aided signal shaping methods in [38]. Also, we investigate the proposed CBSS approach in large-scale systems and compare it with the sole spatial constellation design. In the last subsection, we investigate the impact of channel uncertainty on the error performance. 
TABLE II

Minimum Euclidean Distance Comparisons in $(3,2,2,3)$ GenSM MIMO SYSTEMS

\begin{tabular}{|c|c|c|c|}
\hline- & $\delta=0$ & $\delta=0.1$ & $\delta=0.3$ \\
\hline BPSK & 1 & 0.7007 & 0.4850 \\
\hline OBSS & 1.4768 & 1.5738 & 1.6508 \\
\hline
\end{tabular}

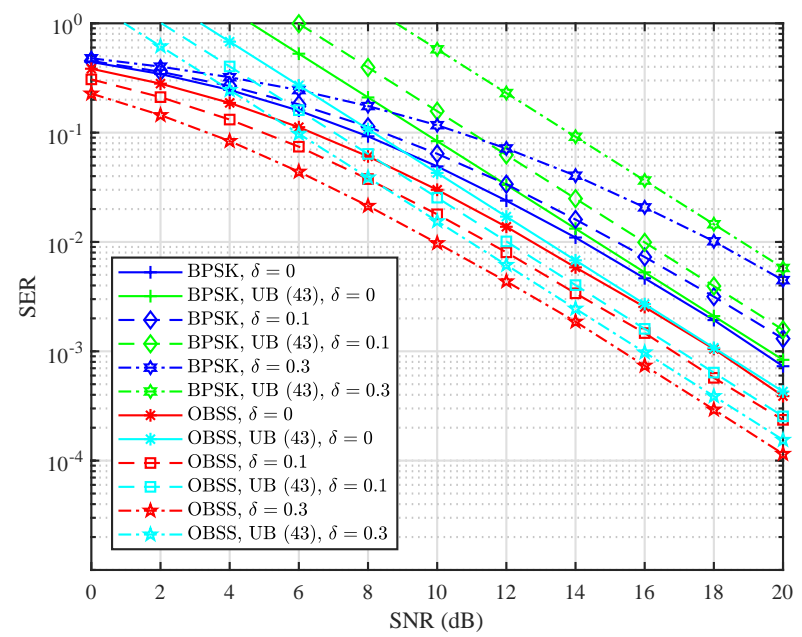

Fig. 3. SER comparisons in $(3,2,2,3)$ GenSM MIMO systems with no $\operatorname{CSIT}(\delta=0) /$ with statistical CSIT $(\delta=0.1,0.3)$

\section{A. Superiority of the Proposed Design in Open-Loop Systems without CSIT and Closed-Loop Systems with Statistical CSIT}

Firstly, we compare the proposed OBSS design with the well-recognized optimal signal constellation in $(3,2,2,3)$ GenSM MIMO systems under different channel conditions. In $(3,2,2,3)$ GenSM systems, two TACs selected from all $\left(\begin{array}{l}3 \\ 2\end{array}\right)=3$ ones can provide a rate of 1 bpcu, subtracting which we can derive that binary modulation is used for 2-datastream carrying $2 \mathrm{bpcu}$. The BPSK as the optimal binary signal constellation is adopted for comparison. We compare the optimized signal shaping with BPSK-based shaping in terms of the minimum Euclidean distances $d_{\min }\left(\mathcal{X}_{8}, \mathbf{R}\right)$ as listed in Table II. It is found that the proposed OBSS optimization strategy has a much larger $d_{\min }\left(\mathcal{X}_{8}, \mathbf{R}\right)$ for $\delta=0, \delta=0.1$ and $\delta=0.3$ and as $\delta$ increases, $d_{\min }\left(\mathcal{X}_{8}, \mathbf{R}\right)$ also increases. The SER comparisons are illustrated in Fig. 3, where the analytical upper bounds presented in (43) are also included. It demonstrates that OBSS outperforms $(3,2,2,3)$ GenSM with BPSK by $1 \mathrm{~dB}$ over independent Rayleigh channels (i.e., $\delta=0$ ). The gain is achieved at no expense. Over the transmitcorrelated channels, results show that the OBSS approach can bring a higher gain. Specifically, more than $3.5 \mathrm{~dB}$ and 8 $\mathrm{dB}$ are achieved at an SER of $10^{-2}$ when $\delta=0.1$ and 0.3 respectively owing to the joint optimization of spatial and signal constellation.

To show more results, we also make the comparisons regarding SER in $(4,2,2,4)$ GenSM MIMO systems where BPSK is also employed for comparison. Fig. 4 demonstrates similar trends that more than $1 \mathrm{~dB}, 3 \mathrm{~dB}$ and $6 \mathrm{~dB}$ are achieved compared to GenSM with BPSK when $\delta=0,0.1$

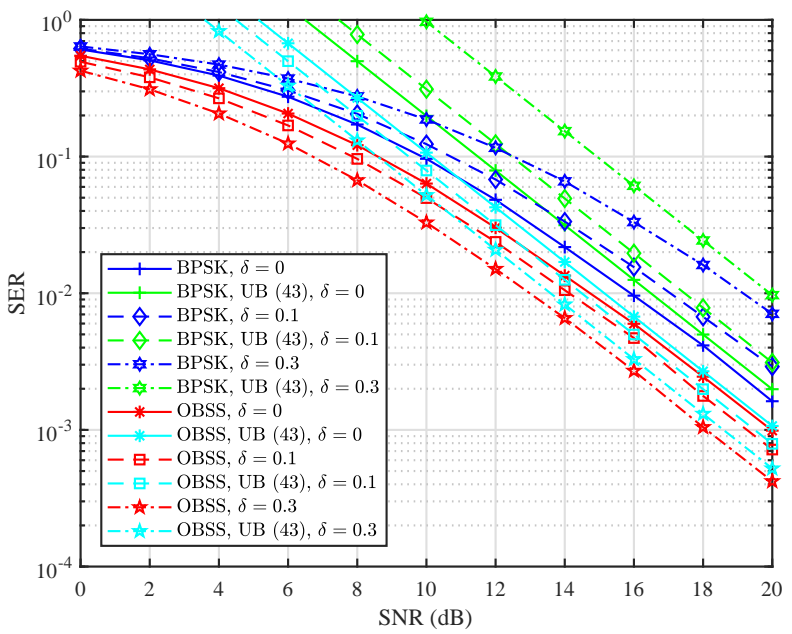

Fig. 4. SER comparisons in $(4,2,2,4)$ GenSM MIMO systems with no CSIT $(\delta=0) /$ with statistical CSIT $(\delta=0.1,0.3)$

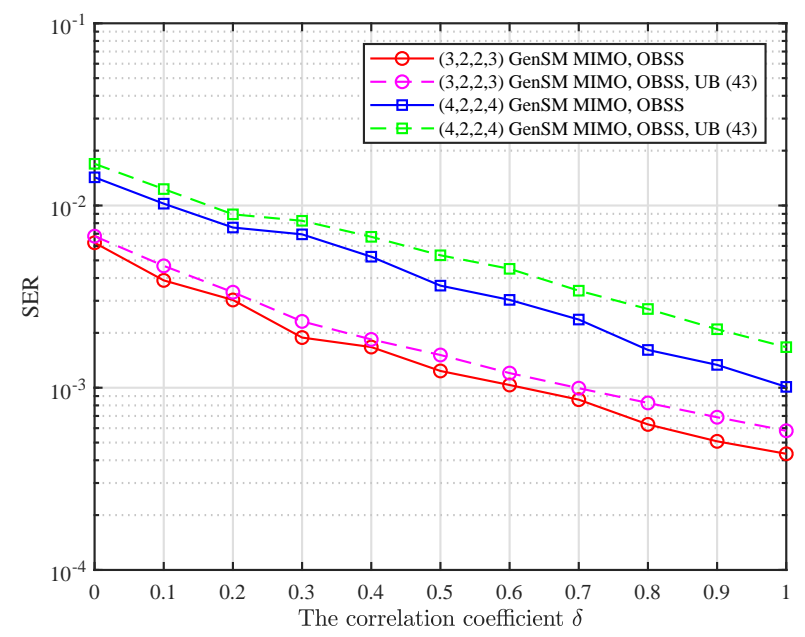

Fig. 5. SER performance at an SNR of $14 \mathrm{~dB}$ with respect to the correlation coefficient $\delta$ ranging from 0 to 1 .

and 0.3 , respectively. All above comparisons show that the OBSS approach can be used to combat transmit correlation and can even benefit from the transmit correlation. Also, we present the SER performance at an SNR of $14 \mathrm{~dB}$ in highlycorrelated systems, where the transmit correlation coefficient $\delta$ is set to range from 0 to 1 as depicted in Fig. 5. The results show that the SER performance of OBSS gets better as $\delta$ increases under the given system setups. The reason behind this is that even through the correlation increases the overlap between the column subspaces, it enlarges these subspaces. For general $\left(N_{t}, N_{r}, N_{R F}, n\right)$ GenSM MIMO systems with OBSS, whether the correlation is always good is still an open question, because the exact relationship between $d_{\min }\left(\mathcal{X}_{N}, \mathbf{R}\right)$ (which determines the SER performance in the high SNR regime) and $\mathbf{R}$ is mathematically unknown for general $\left(N_{t}, N_{r}, N_{R F}, n\right)$ GenSM MIMO systems. But for some specific systems, the correlation can be proved to be good, e.g., the $(2,2,1,1)$ SM MIMO system with the square root of correlation matrix given 
TABLE III

Minimum Euclidean Distance Comparisons in $(3,2,2,4)$ GenQSM MIMO SYSTEMS

\begin{tabular}{|c|c|c|c|}
\hline- & $\delta=0$ & $\delta=0.1$ & $\delta=0.3$ \\
\hline$\frac{\pi}{4}$-BPSK & 0.7071 & 0.4954 & 0.3430 \\
\hline OBSS & 1.2852 & 1.2754 & 1.2614 \\
\hline
\end{tabular}

by

$$
\hat{\mathbf{R}}_{t x}^{1 / 2}=\left[\begin{array}{cc}
1 & \sqrt{\delta} \\
\sqrt{\delta} & 1
\end{array}\right],
$$

in which one of the exact optimal complex vector sets $\hat{\mathcal{X}}_{2}$ can be obtained as

$$
\hat{\mathcal{X}}_{2}=\left\{\hat{\mathbf{x}_{1}}, \hat{\mathbf{x}_{2}}\right\}=\left\{\left[\begin{array}{l}
1 \\
0
\end{array}\right],\left[\begin{array}{c}
-1 \\
0
\end{array}\right]\right\} .
$$

Based on these, we can calculate

$$
d_{\min }\left(\mathcal{X}_{2}, \mathbf{R}\right)=d_{\min }\left(\hat{\mathcal{X}}_{2}, \hat{\mathbf{R}}_{t x}^{1 / 2}\right)=2 \sqrt{1+\delta},
$$

which increases as $\delta$ increases. Additionally, we note that the price paid to achieve such substantial gains in the transmit correlated systems is just the knowledge of $\delta$. Knowing $\delta$ is sometimes costless as $\delta$ can be simply determined by the antenna deployment.

Secondly, to validate the performance superiority of the proposed optimization strategies in GenQSM systems, we make the minimum Euclidean distance and SER comparisons in $(3,2,2,4)$ GenQSM MIMO systems. Since the original BPSK cannot be directly applied to GenQSM MIMO systems because of the zeros in the imaginary parts, we use a $\frac{\pi}{4}$ phaserotated BPSK as the symbol modulation for GenQSM and the rotation does not change its optimality. The comparison results in terms of minimum Euclidean distance and SER are presented in Table III and Fig. 6, respectively. It is observed from Table III that the proposed OBSS approach can also bring considerable performance improvement in maximizing the minimum Euclidean distances when applied to GenQSM systems. For $\delta=0,0.1$ and 0.3 , the optimized minimum Euclidean distances are almost the same. SER comparisons in Fig. 6 demonstrate that when $\delta=0.3$ the systems achieves a lower SER. It is inconsistent with the minimum Euclidean distance comparisons. The reason is that the SER is determined not only by the minimum Euclidean distance but also by the other pairwise Euclidean distances (c.f., (43)). Compared with GenQSM using $\frac{\pi}{4}$-BPSK, the proposed OBSS approaches bring substantial performance improvements by about $2 \mathrm{~dB}, 5 \mathrm{~dB}$ and $9 \mathrm{~dB}$ for $\delta=0,0.1$ and 0.3 , respectively. This validates the performance superiority of our design in GenQSM systems.

\section{B. Superiority of the Proposed Designs in Closed-Loop Sys- tems with Instantaneous CSIT}

With perfect CSIT, we investigate the proposed designs in $(3,2,2,3)$ GenSM MIMO systems, we plot the complementary cumulative distribution function (CCDF) of the minimum Euclidean distance $d_{\min }\left(\mathcal{X}_{8}, \mathbf{H}\right)$ in Fig. 7. The probability $\operatorname{Pr}\left\{d_{\min }\left(\mathcal{X}_{8}, \mathbf{H}\right)>1.5\right\}$ for GenSM with BPSK, GenSM with

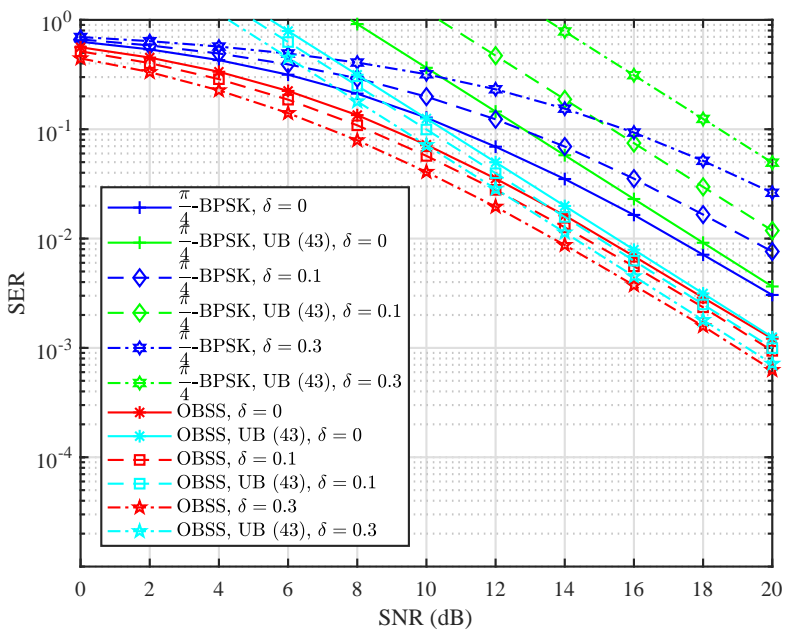

Fig. 6. SER comparisons in $(3,2,2,4)$ GenQSM MIMO systems with no CSIT $(\delta=0) /$ with statistical CSIT $(\delta=0.1,0.3)$.

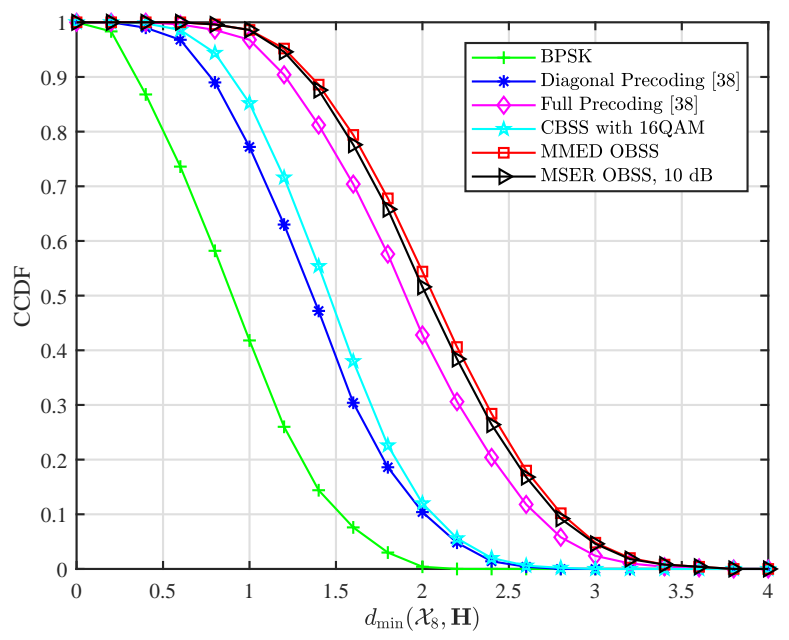

Fig. 7. CCDF comparisons in adaptive $(3,2,2,3)$ GenSM MIMO systems with perfect CSIT.

BPSK and the diagonal precoding proposed in [38], GenSM with BPSK with the full precoding given in [38], GenSM with CBSS and 16QAM as the codebook, GenSM with MMED OBSS, and GenSM with MSER OBSS (at an SNR of $10 \mathrm{~dB}$ ) is $0.1,0.43,0.5,0.8,0.86$ and 0.858 , respectively. From these results, MMED OBSS is the best and the open-loop BPSKbased shaping is the worst. The SER comparisons among these schemes are illustrated in Fig. 8, from which we observe that the proposed OBSS approaches outperforms BPSK with the full precoding and the one with diagonal precoding by around $1 \mathrm{~dB}$ and $4 \mathrm{~dB}$ at an SER of $10^{-2}$, respectively. The proposed CBSS approaches outperforms BPSK with diagonal precoding by more than $2 \mathrm{~dB}$ at an SER of $10^{-2}$. Moreover, as shown in Table I, the CBSS approach is of very low computational complexity compared to other schemes, and it would suit practical implementation in realistic MIMO systems with a limited processing capability. As expected, MSER OBSS slightly outperforms MMED OBSS in terms of SER. Moreover, 


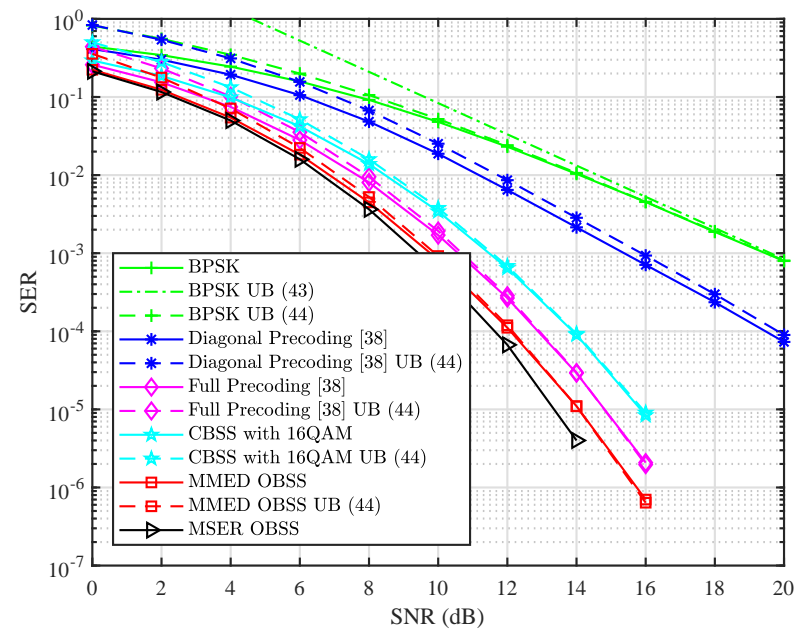

Fig. 8. SER comparisons in adaptive $(3,2,2,3)$ GenSM MIMO systems with perfect CSIT.

we find from the numerical and simulation results in Fig. 8 that the OBSS and CBSS approaches can increase the diversity order. That is, the SER performance of OBSS and CBSS with CSIT decays faster as SNR increases than the shaping without CSIT. However, it should be mentioned that the exact diversity order is unknown, because the signal set $\mathcal{X}_{N}$ with CSIT is obtained according to $\mathbf{H}$ by optimization techniques. As a result, the diversity order $D=\lim _{\rho \rightarrow+\infty} \frac{\mathbb{E}_{\mathbf{H}}\left[\ln \overline{P_{s}}\left(\mathcal{X}_{N}\right)\right]}{\ln \rho}$ is not mathematically tractable.

To show the performance of the proposed signal shaping in large-scale systems, we investigate the adaptive signal shaping in $(10,5,2,7)$ GenSM MIMO systems. The computational complexity burden of the proposed OBSS approach and that of the precoding-based signal shaping proposed in [38] are overwhelming and are infeasible for implementing the adaptive shaping in the given systems. Therefore, we only investigate the low-complexity CBSS approach with 4-QAM as the codebook. For comparison, the SER performance of $(10,5,2,7)$ GSM MIMO systems with BPSK, 4-QAM employed for 2data-stream and fixed/adaptive spatial constellation designs is also included. Results show that the CBSS approach that jointly optimizes the multi-dimensional signal constellation and spatial constellation outperforms the schemes that solely optimize the spatial constellation.

\section{Superiority of the Proposed Designs in the Presence of Channel Estimation Errors}

To show the performance of the proposed designs relying on imperfect CSI, channel estimation errors are considered and modeled as $\hat{\mathbf{H}}_{\mathrm{im}}=\hat{\mathbf{H}}+\hat{\mathbf{H}}_{\mathrm{e}}$, where $\hat{\mathbf{H}}_{\mathrm{im}}$ denotes the estimated channel matrix, $\hat{\mathbf{H}}_{\mathrm{e}} \in \mathbb{C}^{N_{r} \times N_{t}}$ represents estimation error matrix and $\left[\hat{\mathbf{H}}_{\mathrm{e}}\right]_{k, l} \sim \mathcal{C N}\left(0, \sigma_{e}^{2}\right)$. In the training-based channel estimation scheme, letting $E_{p}$ and $N_{p}$ represent the average power and the number of pilot symbols, the variance $\sigma_{e}^{2}=1 /\left(\rho E_{p} N_{p}\right) \triangleq \eta / \rho$ if the least square (LS) channel estimation scheme is adopted [43], [44]. We compare the SER in presence of channel estimation errors for $\eta=0.2$ in Fig. 10.

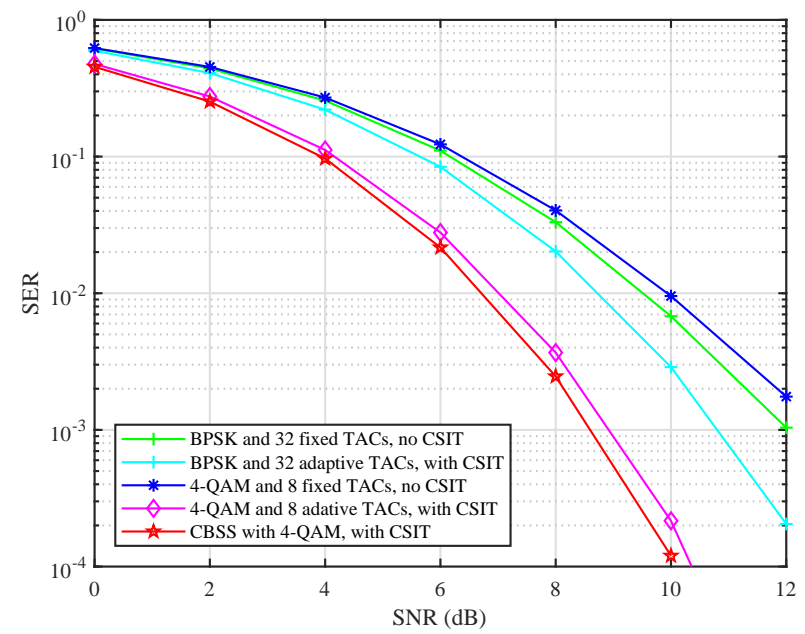

Fig. 9. SER comparisons in adaptive $(10,5,2,7)$ GenSM MIMO systems with perfect CSIT.

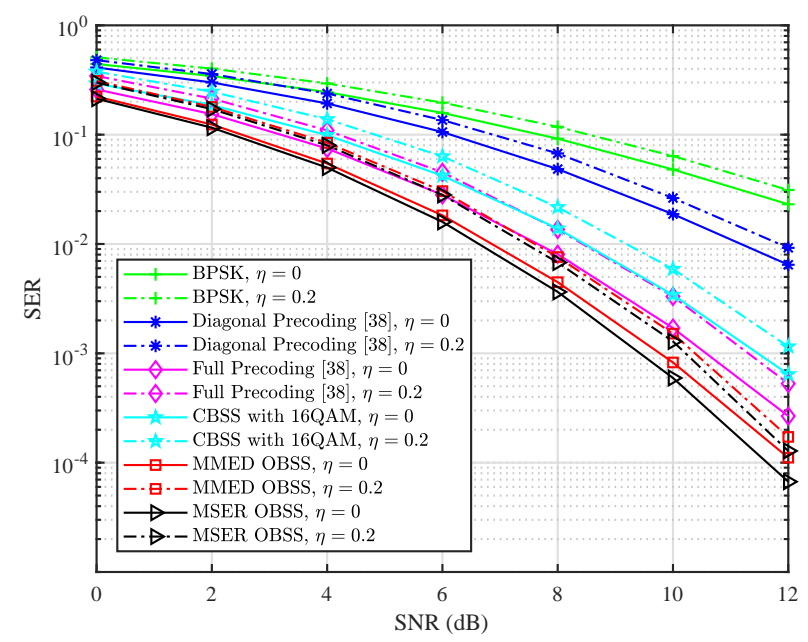

Fig. 10. SER comparisons in adaptive $(3,2,2,3)$ GenSM MIMO systems with perfect and imperfect CSIT.

In the presence of channel estimation errors, the performance of all schemes degrades by a certain level. Despite this, the proposed OBSS approach maintains its championship. Clearly, the performance gains brought by our designs are robust to channel estimation errors.

\section{CONCLUSIONS}

This paper investigated generic signal shaping for multipledata-steam GenSM/GenQSM MIMO systems. A unified optimization problem was formulated. We studied an OBSS approach and a CBSS approach. Results showed that the proposed approaches can be applied to open-loop system$\mathrm{s}$ as well as closed-loop systems to harvest a remarkable performance gain. The OBSS approach exhibited the best performance compared to existing benchmarks. It can also be used to combat the transmit correlation. Simulation results also showed that the OBSS approach can even benefit from transmit correlation to offer performance gains. The CBSS approach 
showed comparable performance but only required quite low complexity. Simulation results validated the superiority of the proposed optimization strategies with perfect and imperfect CSIT.

\section{REFERENCES}

[1] R. Y. Mesleh, H. Haas, S. Sinanovic, C. W. Ahn, and S. Yun, "Spatial modulation," IEEE Trans. Veh. Technol., vol. 57, no. 4, pp. 2228-2241, July 2008.

[2] Y. Yang and B. Jiao, "Information-guided channel-hopping for high data rate wireless communication," IEEE Commun. Lett., vol. 12, no. 4, pp. 225-227, Apr. 2008.

[3] M. D. Renzo, H. Haas, A. Ghrayeb, S. Sugiura, and L. Hanzo, "Spatial modulation for generalized MIMO: Challenges, opportunities, and implementation," Proc. IEEE, vol. 102, no. 1, pp. 56-103, Jan. 2014.

[4] P. Yang, M. D. Renzo, Y. Xiao, S. Li, and L. Hanzo, "Design guidelines for spatial modulation," IEEE Commun. Surveys Tuts., vol. 17, no. 1, pp. 6-26, Firstquarter 2015.

[5] R. Mesleh, S. S. Ikki, and H. M. Aggoune, "Quadrature spatial modulation," IEEE Trans. Veh. Technol., vol. 64, no. 6, pp. 2738-2742, Jun. 2015.

[6] J. Li, M. Wen, X. Cheng, Y. Yan, S. Song, and M. H. Lee, "Generalized precoding-aided quadrature spatial modulation," IEEE Trans. Veh. Technol., vol. 66, no. 2, pp. 1881-1886, Feb. 2017.

[7] M. Mohaisen, "Increasing the minimum Euclidean distance of the complex quadrature spatial modulation," IET Commun., vol. 12, no. 7, pp. 854-860, May 2018.

[8] M. Di Renzo and H. Haas, "Bit error probability of SM-MIMO over generalized fading channels," IEEE Trans. Veh. Technol., vol. 61, no. 3 , pp. 1124-1144, Mar. 2012.

[9] M. Di Renzo and H. Haas, "On transmit diversity for spatial modulation MIMO: Impact of spatial constellation diagram and shaping filters at the transmitter," IEEE Trans. Veh. Technol., vol. 62, no. 6, pp. 2507-2531, July 2013.

[10] P. Yang, Y. Xiao, B. Zhang, S. Li, M. El-Hajjar, and L. Hanzo, "StarQAM signaling constellations for spatial modulation," IEEE Trans. Veh. Technol., vol. 63, no. 8, pp. 3741-3749, Oct. 2014.

[11] P. Zhang, C. Wang, S. Guo, and H. Zhang, "Novel APM constellation design for spatial modulation systems," in Proc. IEEE WCSP, Nanjing, China, Oct. 2015, pp. 1-5.

[12] M. Maleki, H. R. Bahrami, and A. Alizadeh, "Constellation design for spatial modulation," in Proc. IEEE ICC, London, UK, June 2015, pp. 2739-2743.

[13] M. Maleki, H. R. Bahrami, and A. Alizadeh, "Adaptive antenna subset selection and constellation breakdown for spatial modulation," IEEE Commun. Lett., vol. 18, no. 9, pp. 1649-1652, Sept. 2014.

[14] M. Maleki, H. R. Bahrami, A. Alizadeh, and N. H. Tran, "On the performance of spatial modulation: Optimal constellation breakdown," IEEE Trans. Commun., vol. 62, no. 1, pp. 144-157, Jan. 2014.

[15] S. Guo, H. Zhang, S. Jin, and P. Zhang, "Spatial modulation via 3-D mapping," IEEE Commun. Lett., vol. 20, no. 6, pp. 1096-1099, June 2016.

[16] S. Guo, H. Zhang, P. Zhang, D. Wu, and D. Yuan, "Generalized 3D constellation design for spatial modulation," IEEE Trans. Commun., vol. 65 , no. 8, pp. 3316-3327, Aug. 2017.

[17] A. Iqbal, M. Mohaisen, and K. S. Kwak, "Modulation set optimization for the improved complex quadrature SM," Wirel. Commun. Mob. Com. vol. 2018, no. 6769484, pp. 1-12, July 2018.

[18] J. Choi, Y. Nam, and N. Lee, "Spatial lattice modulation for MIMO systems," IEEE Trans. Signal Process., vol. 66, no. 12, pp. 3185-3198, June 2018.

[19] P. Yang, Y. Xiao, L. Li, Q. Tang, Y. Yu, and S. Li, "Link adaptation for spatial modulation with limited feedback," IEEE Trans. Veh. Technol., vol. 61, no. 8, pp. 3808-3813, Oct. 2012.

[20] R. Rajashekar, K. V. S. Hari, and L. Hanzo, "Antenna selection in spatial modulation systems," IEEE Commun. Lett., vol. 17, no. 3, pp. 521-524, Mar. 2013.

[21] K. Ntontin, M. D. Renzo, A. I. Perez-Neira, and C. Verikoukis, "A lowcomplexity method for antenna selection in spatial modulation systems," IEEE Commun. Lett., vol. 17, no. 12, pp. 2312-2315, Dec. 2013.

[22] R. Rajashekar, K. V. S. Hari, and L. Hanzo, "Quantifying the transmit diversity order of euclidean distance based antenna selection in spatial modulation," IEEE Signal Process. Lett., vol. 22, no. 9, pp. 1434-1437, Sept. 2015.
[23] M. D. Renzo and H. Haas, "Improving the performance of space shift keying (SSK) modulation via opportunistic power allocation," IEEE Commun. Lett., vol. 14, no. 6, pp. 500-502, June 2010.

[24] M. Maleki, H. R. Bahrami, S. Beygi, M. Kafashan, and N. H. Tran, "Space modulation with CSI: Constellation design and performance evaluation," IEEE Trans. Veh. Technol., vol. 62, no. 4, pp. 1623-1634, May 2013.

[25] Y. Xiao, Q. Tang, L. Gong, P. Yang, and Z. Yang, "Power scaling for spatial modulation with limited feedback," International Journal of Antennas and Propagation, vol. 2013, pp. 1-5, June 2013.

[26] S. Guo, H. Zhang, J. Zhang, and D. Yuan, "On the mutual information and constellation design criterion of spatial modulation mimo systems," in Proc. IEEE ICCS, Nov. 2014, pp. 487-491.

[27] J. Zhang, Q. Li, K. J. Kim, Y. Wang, X. Ge, and J. Zhang, "On the performance of full-duplex two-way relay channels with spatial modulation," IEEE Trans. Commun., vol. 64, no. 12, pp. 4966-4982, Dec. 2016.

[28] P. Yang, Y. Xiao, B. Zhang, S. Li, M. El-Hajjar, and L. Hanzo, "Power allocation-aided spatial modulation for limited-feedback MIMO systems," IEEE Trans. Veh. Technol., vol. 64, no. 5, pp. 2198-2204, May 2015.

[29] L. He, J. Wang, C. Zhang, and J. Song, "Improving the performance of spatial modulation by phase-only pre-scaling," in Proc. IEEE ICC, London, UK, June 2015, pp. 3210-3215.

[30] M. Lee, W. Chung, and T. Lee, "Generalized precoder design formulation and iterative algorithm for spatial modulation in MIMO systems with CSIT," IEEE Trans. Commun., vol. 63, no. 4, pp. 1230-1244, Apr. 2015.

[31] P. Yang, Y. L. Guan, Y. Xiao, M. D. Renzo, S. Li, and L. Hanzo, "Transmit precoded spatial modulation: Maximizing the minimum Euclidean distance versus minimizing the bit error ratio," IEEE Trans. Wireless Commun., vol. 15, no. 3, pp. 2054-2068, Mar. 2016.

[32] J. Zhang, Y. Wang, J. Zhang, and L. Ding, "Polarization shift keying (PolarSK): System scheme and performance analysis," IEEE Trans. Veh. Technol., vol. 66, no. 11, pp. 10139-10155, Nov. 2017.

[33] W. Wang and W. Zhang, "Diagonal precoder designs for spatial modulation," in Proc. IEEE ICC, London, UK, June 2015, pp. 2411-2415.

[34] _ - "Adaptive spatial modulation using Huffman coding," in IEEE GLOBECOM, Washington, DC USA, Dec. 2016, pp. 1-6.

[35] — , "Huffman coding-based adaptive spatial modulation," IEEE Trans. Wireless Commun., vol. 16, no. 8, pp. 5090-5101, Aug. 2017.

[36] _ - "Transmit signal designs for spatial modulation with analog phase shifters," IEEE Trans. Wireless Commun., vol. 17, no. 5, pp. 3059-3070, May 2018.

[37] N. S. Perovic, P. Liu, J. Blumenstein, M. Di Renzo, and A. Springer, "Optimization of the cut-off rate of generalized spatial modulation with transmit precoding," IEEE Trans. Commun., vol. 66, no. 10, pp. 45784595, Oct. 2018.

[38] P. Cheng, Z. Chen, J. A. Zhang, Y. Li, and B. Vucetic, "A unified precoding scheme for generalized spatial modulation," IEEE Trans. Commun., vol. 66, no. 6, pp. 2502-2514, June 2018.

[39] R. Sheldon, A First Course in Probability, 8 Ed. Englewood Cliffs, New Jersey, USA: Prentice-Hall, 2010.

[40] W. Wang and W. Zhang, "Signal shaping and precoding for MIMO systems using lattice codes," IEEE Trans. Wireless Commun., vol. 15, no. 7, pp. 4625-4634, July 2016.

[41] W. Zeng, C. Xiao, and J. Lu, "A low-complexity design of linear precoding for MIMO channels with finite-alphabet inputs," IEEE Wireless Communications Letters, vol. 1, no. 1, pp. 38-41, Feb. 2012.

[42] T. Handte, A. Muller, and J. Speidel, "BER analysis and optimization of generalized spatial modulation in correlated fading channels," in 2009 IEEE 70th VTC Fall. Anchorage, AK, USA: IEEE, Sept. 2009, pp. $1-5$.

[43] S. Guo, H. Zhang, P. Zhang, and D. Yuan, "Link-adaptive mapper designs for space-shift-keying-modulated MIMO systems," IEEE Trans. Veh. Technol., vol. 65, no. 10, pp. 8087-8100, Oct. 2016.

[44] S. Dang, J. P. Coon, and G. Chen, "Adaptive OFDM with index modulation for two-hop relay-assisted networks," IEEE Trans. Wireless Commun., vol. 17, no. 3, pp. 1923-1936, Mar. 2018. 